\title{
Nicos Poulantzas Das Problem des kapitalistischen Staates
}

$[\ldots]$

(Ehe sich P. mit dem Buch von Miliband auseinandersetzt, untersucht er kurz die Ursachen des Fehlens einer marxistischen Staatstheorie; als wesentliche Ursache erscheine der Okonomismus der $Z$ weiten und Dritten Internationale.)

In diesem Zusammenhang trägt Milibands Buch $»$ The State in Capitalist Society " ${ }^{1}$ dazu bei, eine große Lücke zu schließen. Wie es immer der Fall ist, wenn keine wissenschaftliche Analyse vorliegt, haben auch hier bürgerliche Ideologien über den Staat und die politische Macht beinahe unangefochten das Feld der politischen Theorie beherrsche. Die Arbeit Milibands hat in dieser Beziehung kathartische Wirkung: systematisch tritt er diesen Ideologien entgegen. In seiner Untersuchung der konkreten Gesellschaftsformen in den USA, England, Frankreich, Deutschland oder Japan breitet er sorgfältig eine ungeheure Fülle von empirischem Material aus und zerstört damit nicht nur radikal die bürgerlichen Ideologien vom Staat, sondern liefert ein posirives Wissen, das hervorzubringen diese Ideologien niemals in der Lage gewesen sind. Dennoch ist das von Miliband gewählte Verfahren - eine direkte Antwort auf bürgerliche Ideologien durch unmittelbare Analyse der konkreten Fakten - meiner Auffassung nach zugleich Ursache der Fehler scines Buches. [...] Es ist in diesem Zusammenhang wichtig festzustellen, daß Miliband an keiner Stelle die marxistische Staatstheorie als solche behandelt, obgleich sie ständig in seinem Werk implizit enthalten ist. Esetzt sie einfach als $>$ gegeben voraus, um durch die Untersuchung der Fakten in ihrem Lichte den bürgerlichen Ideologien entgegenzutreten. Meiner festen Uberzeugung nach irrt Miliband hier, denn das Fehlen einer expliziten Darlegung der Grundsätze bei einer wissenschaftlichen Argumentation bleibr nicht ohne Folgen: vor allem auf einem Gebier wie der Stasstheorie, wo eine marxistische Theorie erst noch erarbeitet werden muß. Man hat in der Tat den Eindruck, daß dieser Mangel Miliband of veranlaßt, die bürgerlichen Ideologien vom Staate anzugreifen und sich dabei selbst auf deren Boden zu begeben. Statt

\footnotetext{
* Anmerksng zur Ubersetzung:

Der Ubersetzung lagen der Text van Nicos Poulantzas, The Problem of the Capitalist Statee in: New Left Review 1969, Nr. 58, S. 67-78, und die Erwiderung von Ralph Miliband, -The Capitalist State: Reply to Nicos Poulanizase, in: New Left Review 1969, Nr. 59, S. s3-6o zugrunde. Poulantzas hat seinen Text späzer in z. T. erweiterter und veränderter Fassung in französisch emcut publiziert unter dem Tizel sur l'Etat dans la sociéré capitaliste. in: politique aujourd'hui, mars 1970 , S. 6s-76. Soweit der französische vom englischen Text wesentlich abweichs, warden die Abwerchungen aufgejührt und durch [p. a.:...] gekennzeiojnet. Die Ubersetzung besorgten Klaus Lenk, Ulrich Mückenberger und Joachim Perels.

Beide Texte mußten aus Platzgründen gekürzt uerden. Die Auslassungen sind mit eckigen Klammern $[.$. ] bezeschnet.

1 Weidenield and Nicholson, London 1969.
} 
den erkenntnistheoretischen Ausgangspunkt zu verwerfen und diese Ideologien durch den Nachrweis ihrer Inadäquatheit in Bezug auf die Realitär der Kritik marxistischer Wissenschaft zu unterwerfen (wie Marx es tur, insbesondere in den Theorien ïber den Miebrwert), scheint Miliband diesen ersten Schritt auszulassen. [...]

Nehmen wir ein einfaches Beispiel. Miliband greift die vorherrschende Vorstellung eines $*$ Pluralismus von Eliten* an, deren ideologische Funktion es ist, die Existenz einer herrschenden Klasse zu negieren und wendet - auf $\rightleftharpoons$ Fakten $\propto$ gestützt - ein, daß dieser »Pluralismus von Eliten dic Existenz einer herrschenden Klasse nicht ausschließ̊t, weil es gerade diese Eliten seien, die diese Klasse konstituieren²; das entspricht ungefähr der Auffassung Bottomores in dieser Frage. [...] Was Miliband vermissen läßt, ist die notwendige Vorstufe [p.a.: - das - logische Prius “, sagte Marx -] einer Kritik der ideologischen Kategorie der Elite im Licht der wissenschaftlichen Konzepte marxistischer Theorie. Wäre diese Kritik geliefert worden, so wäre offenbar geworden, daß die Vorstellung eines "Pluralismus von Eliten « - die herrschende Klasse und deren Fraktionen, die hegemoniale Klasse, die regierende Klasse, der Staatsapparat - die skonkrete Realitüt« verschlciert und daß diese nur e:faßt werden kann, wenn genau dic Kategorie der Elite zurückgewiesen wird. Denn Konzepte und Begriffe sind niemals »rein «, und durch die Ubernahme der Begriffe des Gegners legitimiert und verlängert man nur deren Geitung. Alle Begriffe und Konzepte haben ihre Bedeutung nur innerhalb des sie begründenden theoretischen [p.a.: und politischen] Problemzusammenhanges: reißt man sie aus diesem Problemzusammenhang heraus und übernimme sie wunkritisch « in die marxistische Theoric, so hat das unabsehbare Foigen [p. a.: man denke etwa an den Siandardbegriff $\times$ bürgerliche Gesellschaft«]. Sie tauchen immer auf, wo man sie am wenigsten erwarter, und drohen die wissenschaftliche Analyse zu überschatten. Im Extremfall macht man sich unbewußt und unmerklich gerade die erkenntnistheoretischen Grundsätze des Gegners zu eigen, also den Problemzusammenhang, der seine Konzepte begründet, die man nicht theoretisch kritisiert hat, weil man annahm, sie wären bereits von den $\bowtie$ Fakten $*$ widerlegt. Das wiegt noch schwerer: denn dann wezden nicht mehr nur fremde Kategorien in den Marxismus whineingetragen «, sondern Grundsätze, die die Anwendung marxistischer Konzepte selbst zu beeinträchtigen drohen. [...] Wie wird das deutlich? Kurz gesagt: in den Schwierigkeiten, die Miliband hat, soziale Klassen und den Staat als objektive Strukturen zu begreifen und ihr Verhältnis als ein objektives $S_{y}$ stem gese:zmäßiger Beziehungen, eine Struktur und ein System, deren Agenten, "Menschen ", in den Worten von Marx, -Trägere sind. Man hat ständig den Eindruck, daß für Miliband soziale Klassen oder „Gruppen« irgendwie reduziert werden können auf interpersonale Beziénungen; daß der Staat reduziert werden kann auf die interpersonalen Beziehungen der Mitglieder der vezschiedenen Gruppen $\propto$, die den Staatsapparat konstituieren, und daß schließlich die Beziehungen $z$ wischen sozialen Klassen und Staat selbst reduziert werden können auf interpersonale Beziehungen von sIndividuen ", die soziale Gruppen biiden, und sIndividuen $\alpha$, die den Staatsapparat bilden.

$[\ldots]$

Wir wollen nun einige konkrete Themen von Milibands Buch im Lichte dieses Vorspanins betrachten.

2 Miliband, S. 24 ff. und 47 . 
Selir zutreffend diskutiert Miliband als erstes Problem das der sherrschenden Klassee, wobei er den gängigen bürgerlichen Ideologien von der Managerberrschaft entgegentritt. Nach diesen Ideologien hat das gegenwärtige Auseinanderfallen von Eigentum und Kontrolle die ökonomische Macht von den Kapitalisten auf die Manager übergehen lassen. Letztere hätten keine Eigentümerinteressen im strengen Sinne, wären nicht primär am Profit interessiert - mit anderen Worten, nicht Profit sei ihr Handlungsziel, sondern Wachstum und Entwicklung [p. a.: »industrielle Entwicklung «, »wirtschaftliche Stärke« etc.]. Da die herrschende Klasse dabei durch Gewinnstreben definiert wird und dieses Streben nicht mehr die heutigen Wirsschaftsführer charakterisiert, gibt es die herrschende Klasse selbst nicht mehr: wir stehen jetzt einem "Pluralismus von Eliten " gegenüber, deren eine die Manager sind. Wie antwortet Miliband?3 Er nimmt die Idcologie beim Wort und wendet deren eigene Argumente gegen sie: tatsächlich ist das Verhalten doch durch das Streben nach Profit bestimmt, denn schließlich ist das das Bewegungsgesetz des Kapitalismus. In der Verfolgung des Ziels der Vermehrung privaten Profits sind die Manager Teil der herrschenden Klasse, denn - so belehrt uns Miliband - Marx zufolge ist der Widerspruch des kapitalistischen Systems der w Widerspruch zwischen seinem in immer höherem Maße gesellschaftlichen Charaktez und seiner fortdauernden privaten Zielsetzung «. ${ }^{4}$ Ohne die Existenz mancher für Manager spezifischen Zielsetzungen, die sich von denen der Eigentümer relativ unterscheiden, auszuschließen, betrachtet Miliband Manager als eine unter verschiedenen wirtschaftlichen Eliten, die in ihrer Gesamtheit die herrschende Klasse ausmachen.

Ich halte das für einen falschen Ansatz. Zunächst einmal: Das entscheidende Kriterium für die Zugehörigkeit zur Klasse der Kapitalisten ist für Marx keineswegs eine Verhaltensmotivation, also das Streben nach Gewinn als »Handlungsziel «. Denn es mag sehr wohl Kapitalisten geben, die sich nicht vom Profitstreben bestimmen lassen, genauso wie es Nichtkapitalisten gibt (etwa die Kleinbürger im Kleingewerbe), die genau diese Motivation haben. Marx' Kriterium ist die objektive Stellung im Produktionsprozeß und das Eigentum an Produktionsmitteln. Es ist daran zu erinnern, daß selbst Max Weber zugestehen mußte, nicht das Streben nach Gewinn definiere den Kapitalisten. Für Marx ist der Profit kein Handlungsmotiv - nicht einmal ein vom System »aufgezwungenes «-, sondern er ist eine objektive Kategorie, die einen Teil des realisierten Mehrwerts bezeichnet. Auch ist der Grundwiderspruch des kapitalistischen Systems - Marx zufolge - keineswegs der $z$ wischen seinem gesellschaftlichen Charakter und seiner »privaten Zielsetzung ", sondern der Widerspruch zwischen der Vergesellschaftung der Produktivkräfte und deren privater Aneignung [p. a.: zwischen der fortschreitenden Vergesellschaftung des Produktionsprozesses und der privaten Aneignung der Produktionsmittel]. Deshalb hängt die Charakterisierung des bestehenden sozialen Systems - also des kapitalistischen Systems - in keiner Weise von der Motivation der Tätigkeit der Manager ab.

Ferner: um die Klassenlage der Manager zu charakterisieren, muß man nicht auf die Motivationen ihrer Tätigkeit, sondern lediglich auf ihre Stellung im Produktionsprozeß und ihre Beziehung zum Eigentum an den Produktionsmitteln zurückgreifen. Dabei ist [...] bei dem Begriff »Eigentum«, wie Marx ihn ver-

s Miliband, ebenda.

4 Miliband, S. 34. 
wendet [ $p$. a.: mit dem Marx die Beziehung des Nicht-Arbeiters zu den Produktionsmittcln bezeichnct], zu unterschciden zwischen dem formalrechtlichen Eigentum, das dem »individueilen « Kapitalisten niche zu gehören braucht, und dem ökonomischen Eigentum oder der realen Aneignung, was einzig ökonomische Macht erzeugt. ${ }^{5}$

Dieses ökonomische Eigentum, auf das es bei der Einteilung in Klassen ankommt, ist in der Tat in der Hand des Kapitals. Dem Manager ist es nur der Funktion nach delegiert.

Unter diesem Gesichtspunkt konstituieren die Manager keine gesonderte Fraktion der Kapitalistenklasse, während Miliband aufgrund der nichtstichhaltigen Unterscheidung nach Verhaltensmotiven zu dem Schluß gelangt, die Manager wären als eine besondere "wirtschaftliche Elite « zu betrachten. Damit mißt er ihnen nicht nur eine Bedeutung zu, die sie nicht besitzen, sondern er verliert das Wesentliche aus den Augen. Denn nicht auf Unterschiede und Beziehungen zwischen $»$ wirtschaftlichen Eliten $*$ mit divergierenden Zielvorstellungen kommt es an, sondern auf etwas, worüber Miliband so gut wie nichts sagr: die Unterschiede und Beziebungen zwischen den Fraktionen des Kapitals. Das Problem ist nicht das einer Mehrzahl "wirtschaftlicher Eliten «, sondern das einer Mehrzahl von Fraktionen der Kapitalistenklasse. Kann ein Marxist die im Zeichen des Imperialismus bestehenden Unterschiede und Beziehungen zwischen Monopolkapital, nichtmonopolistischem Kapital, Industriekapital und Finanzkapital schlicht übergehen?

\section{Das Problem des Staatsapparates und der $>$ Bürokratiea}

Nunmehr wender Miliband sich - einleuchtenderweise - dem Problem der Beziehung $\mathrm{z}$ wischen herrschender Klasse und Staat zu: Er geht hier in derselben Weise vor, indem er die bürgerlichen Ideologien direkt zu widerlegen versucht. Diese Ideologien behaupten, der Staat sei als Vertreter des Gemeinwohls neutral gegenüber den divergenten Interessen der »bürgerlichen Gesellschaft«. Manche (wie etwa R. Aron) behaupten, die Bourgeoisie habe in den kapitalistischen Gesellschaften niemals selbst regiert, in dem Sinne, daß ihre Mitglieder nur selten unmittelbar an der Regierung beteiligt gewesen seien. Andere wiederum führen an, die Mitglieder des Staatsapparates, die Beamten, seien neutral gegenüber den Interessen der gesellschaftlichen Gruppen. Auch hier läß sich Miliband dazu verleiten, die genaue Gegenposition einzunehmen, den Spieß einfach umzudrehen. Er tur das in doppelteter Weise. Zunächst weist er nach, daß die Mitglieder der Kapitalistenklasse häufig sehr wohl am Staatsapparat und an der Regierung beteiligt gewesen sind. ${ }^{6}$ Nachdem er die Beziehung zwischen Staatsapparat und herrschender Klasse hergestellt hat, zeigt er, daß a) die soziale Herkunft von Mitgliedern der "Spizze des Staatsapparates die herrschende Klasse ist und b) persönliche Beziehungen durch Einfluß, Status, Milieu etc. zwischen den Mirgliedern der herrschenden Klasse und denen des Staatsapparates bestehen. ${ }^{7}$

Ich habe nicht die Absicht, den Wert der Untersuchungen Milibands zu bestreiten, die mir im Gegenteil von zentraler entscbleiernder Bedeutung zu sein schei-

\footnotetext{
5 Bctrlcheim, La Transition yers l'Economie Socialiste, und Poulantzas, Pouyoir polirique et classe, sociales, Maspero, Paris i 968. S. 23 ff.

- Miliband. S. $4^{8-68 .}$

7 Ebenda, S. $69-145$ (bes. S. IIg-145).
} 
nen. Dennoch halte ich die Methode Milibands, so exakt sie in sich sein mag, nicht für die bedeutsamste. Dies vor allem, weil die unmıttelbare Teilhabe der Kapitalistenklasse an Staatsapparat und Regierung, auch wo es sie gibr, nicht den entscheidenden Punkt ausmacht. Das Verhältnis der bürgerlichen Klasse zum Staat ist ein objektives. Das heißt: Wenn die Funktion des Staates in einer bestimmten sozialen Formation sich mit den Interessen der herrschenden Klasse in dieser Formation deckt, so geschieht dies auf Grund des Systems selbst: die unmitrelbare Bereiligung der Mitglieder der herrschenden Klasse am Staatsapparat ist nicht die Ursache, sondern die - im übrigen zufällige - Folge dieser objektiven Ubereinstimmung.

Um diese objektive Ubereinstimmung nachzuweisen, wäre es nötig gewesen, die Rolle des Staates als einer spezifischen Instanz des gesellschaftlichen Ganzen zu zeigen. Miliband hingegen scheint die Rolle des Staates auf die Verhaltensstrukturen der Mitglieder des Staatsapparates zurückführen zu wollen. ${ }^{8}$ Hätre er gezeigt, daß der Staat genau der Agent des Zusammenhalts und der Reproduktion der Produktionsbedingungen eines Systems ist, das selbst die Herrschaft einer Klasse über die anderen bewirkt, dann wäre ihm klar geworden, daß die Beteiligung dieser $\mathrm{K}$ lasse an der Regierung, ob nun direkt oder indirekr, nichts ändert. Was den kapitalistischen Staat angeht, so kann man noch weitergehend sagen: der kapitalistische Staat [p. a.: mit dem ideologischen Apparat, der ibn kennzeichnet] wird den Interessen der kapitalistischen Klasse am weitestgehenden gerecht, wenn die Mitglieder dieser Klasse nicht direkt an den Staatsgeschäften beteiligt sind, d. h. wenn die herrschende Klasse nicht auch die politisch regierende Klasse ist. Das ist der genaue Inhalt der Analysen von Marx [p.a.: und Engels] über das England des 19. Jahrhunderts und Deutschland unter Bismarck [p.a.: wo der Grundadel regierende Klasse blieb], vom französischen Bonapartismus gar nicht zu reden. Miliband scheint übrigens in seiner Analyse sozialdemokratischer Regierungen diesen Sachverhalt anzudeuten. ${ }^{\ominus}$

Wir wenden uns nun dem Problem der Mitglieder des Staatsapparates zu, d. h. der Armee, Polizei, Justiz und Verwaltung. In seiner Argumentationskette versucht Miliband die Beziehungen zwischen den Interessen der herrschenden Klasse und dem Verhalten der Mitglieder des Staatsapparates dadurch herzustellen, daß er entweder die soziale Ferkunft der "Spitzenbeamten « aus der herrschenden Klasse oder aber die persönlichen Beziehungen nachweist, die die Mitglieder des Staatsapparates schließlich mit der herrschenden Klasse vereint. ${ }^{10}$ Dieser nicht unbedingt falsche Ansatz bleibt völlig deskriptiv. Mehr noch: er verhindert die Analyse eines der spezifischen Probleme des Staatsapparates, des Problems der Bürokratie . Marx, Engels und Lenin zufolge bilden die Mitglieder des Staatsapparates, die man gewöhnlich als „Bürokratie" im weiteren Sinne bezeichner, eine besondere gesellschaftliche Kategorie, aber keine Klasse. Obwohl die Mitglieder des Staatsapparates herkunftsmäßig verschiedenen Klassen [p.a.: und Klassenfraktionen] angehören, fungieren sie entsprechend einem spezifischen Systemzusammenhang. Ihre klassenmäßige Herkunft tritt hinter dem zurück, was sie zusammenhält - ihrer Klassenfunktion: also der Tatsache, daß sie eben zum Staatsapparat gehören und als ihre objektive Funktion die Aktualisierung der Rolle des Staates vorfinden. Das bedeutet umgekehrt, daß die Bürokratie - als eine besondere und relativ »einheitliche soziale Kategorie -

${ }^{3}$ Ebenda, S. 68-Iis.

- Miliband, S. 96 ff.

10 Ebenda, S. Irg-r 45 . 
- Diener der herrschenden Klasse ist, nicht ihrer klassenmäßigen Herkunft, die unterschiedlich ist, oder ihrer persönlichen Beziehungen zur herrschenden Klasse wegen, sondern weil sich ihr Zusammenhalt aus der Aktualisierung der objektiven Rolle des Staates ergibr. Die Totalität dieser Rolle decks sich mit den Interessen der herrschenden Kiasse.

Daraus ergeben sich wichtige Konsequenzen für das oft diskutierte Problem der relativen Autonomie des Staates in bezug auf die herrschende Klasse, und damit für die nicht minder kontroverse Frage nach der relativen Autonomie der Bürokratie als besonderer gesellschaftlicher Kategorie gegenüber dieser Klasse. Eine lange marxistische Tradition sah im Stzat ein einfaches - durch die herrschende Klasse nach Belieben zu manipuliercndes - Werkzeug oder Instrument. Ich will nicht behaupten, daB Miliband in diese Falle geht, die eine genaue Analyse der komplexen Mechanismen des Staates in seinem Verhältnis zum Klassenkampf verhinderc. Aber: Wenn man die Beziehungen zwischen Staatsapparat und herrschender Klasse auf die soziale Herkunft der Mirglieder des Staatsapparates und ihre persönlichen Beziehungen zu Mitgliedern dieser Klasse zurückführt - so, als nähme die Bourgeoisie den Staat physisch »in Besitz « -, kann man der relativen Autonomie des Staztsapparates in bezug auf diese Klasse nicht gerecht werden. Wenn Marx [p. a.: und Engels] den "Bonapartismus« als "Religion der Bourgeoisie" bezeichneten [p.a.: im Unterschied zu ihrer Analyse der konkraten historischen Erscheinung des französischen Bonapartismus], also als für alle Formen des kapitalistischen Staates charakteristisch, wollten sie damit sagen, daB dieser Staat der herrschenden Klasse nur insoweit wirklich von Nutzen sein kann, als er gegenüber deren verschiedenen Fraktionen relativ autonom und eben dadurch in der Lage ist, die Hegemonie der Klasse als Ganzer zu organisieren. [p. a.: In ihren Formen und Modifikationen hängt diese relative Autonomie sicherlich von den unterschiedlichen Formen der kapitalistischen Gesellschaften und Regierungsweisen ab]. Es ist kein Zufall, daß Miliband diese Autonomie schlieBlich nur für den Extremfall des Faschismus zugesteht. ${ }^{11}$

$[\ldots]$

\section{Die $\#$ Weige des Staatsapparates}

$[\ldots]$

(P. geht auf das Verhälenis der einzelnen $>$ Zweigex des Staatsapparates - Regierung, Armee, Polizei, Justiz und Verwaltung - untereinander ein. Keiner dieser $Z$ weige könne isolierr auf seine Bedeurung und Bedeurungsveränderung hin untersucht werden. Dic Bedeutung jedes $Z$ weiges ergebe sich vielmehr aus dem objekriven Systemzusammenhang, in dem er steht, und lasse sich nur durch die Analyse von dessen Veränderungen - dic selbst wieder auf Veränderungen in den Produkrionsverhältnissen und auf den Entwicklungsstufen des Klassenkampfes beruhen - ermitteln.)

\section{Die gegenwiätige Form des kapitalistischen Staates}

$[\ldots]$

(Im Abschnitt 4 geht P. kurz auf die heurige Form des kapitalistischen Staates ein, dem er - entgegen der These vom Staat als schlichter Agentur des Großkapitals - nach wic vor eine relative Autonomie einräume.)

11 Miliband, S. 93. 
$[\ldots]$

Die traditionelle marxistische Staatstheorie hat sich hauptsächlich mit der repressiven Rolle des Staates im Sinne organisierter physischer Repression beschäftigt. Eine bemerkenswerte Ausnalıme macht Gramscis Erörterung des Problems der Hegemonie. Sehr zurecht besteht Miliband in langen und ausgezeichneten Analysen (The Process of Legitimization, I, II, S. 179-264) auf der Rolle, die dem Ideologischen beim Funktionieren des Staates und beim Prozeß politischer Herrschaft zukomme, was ich unter anderem Aspekt in meinem eigenen Buch versucht habe.

Ich glaube allerdings, daß wir beide, wenn auch aus verschiedenen Gründen, auf halbem Wege stehengeblieben sind: was für Gramsci nicht zutrifft.

Wir sind bei der Uberlegung stehengeblieben, Ideologie existiere nur in Ideen, Gewohnheiten oder Sitten, ohne zu sehen, daß Ideologie in einem handgreiflichen Sinne in Institutionen verkörpert sein kann: Institutionen, die dann durch eben den Prozeß der Institutionalisierung zum System des Staates gehören, auch wenn sie prinzipiell dem Bereich der Ideologie zuzurechnen sind. An die marxistische Tradition anknüpfend, neigten wir dazu, den Begriff des Staates sehr eng zu fassen: Die Institurionen mit überwiegend repressiver Funktion betrachteten wir als Teil des Staates und die mit überwiegend ideologischer Funktion verwiesen wir auf einen Ort "außerhalb des Staates, den Miliband als »politisches System « bezeichnet und vom Staat unterschieden wissen will. ${ }^{12}$ Folgende These möchte ich [p. a.: im Anschluß an Louis Althusser ${ }^{13}$ ] aufstellen: das staatliche System besteht aus mebreren Apparaten oder Institutionen, von denen manche eine überwiegend repzessive Rolle [ $p$. a.: im Sinne organisierter physischer Repression], andere eine überwiegend ideologische Funkrion ausüben. Erstere bilden den repressiven Apparat des Staates, also den Staatsapparat im klassischen marxistischen Sinn (Regierung, A-mee, Polizei, Justiz, Verwaltung). Letztere bilden die ideologischen Apparate des Staates, wie die Kirche, die Parteien, Verbände (mit Ausnahme revolutionärer Parteien oder Gewerkschaften), Schulen [p.a.: und Universitäten], Massenmedien ([p.a.: Verlagswesen], Zeitungen, Radio, Fernsehen) und von einer bestimmten Warte aus [p.a.: zumindest unter kapitalistischen Produktionsverhältnissen] auch die Familie. Das gilt unabhängig vom formalen öffentlichen oder privaten Charakter der Institutionen [p. a.: also unabhängig davon, ob sie offiziell und formal »Teil« des Staates sind], eine Unterscheidung, die rein juristisch und damit weithin ideologisch ist und nichts Grundlegendes ändert.

Diese Position ist gewissermaßen die Gramscis, auch wenn er sie nicht hinreichend fundierte und vrciterentwickclte.

Warum sollte man von den ideologischen Staatsapparaten im Plural und von dem (repressiven) Staatsapparat im Singular sprechen? Weil der repressive Staatsapparat, der Staat im klassischen marxistischen Sinn, einen sehr starken Systemzusammenhang besitzr, der unmittelbar das Verhältnis der verschiedenen Zweige des Apparates steuert.

Demgegenüber besitzen die ideologischen Apparate des Staates wegen ihrer Hauptfunktion - Indolstrination und Ubermittlung - eine größere und wichtigere Autonomie: ihre Beziehungen untereinander und zum repressiven Staats-

Is Miliband. S. so ff.

13 Vervielfälrigrer Texr, März-April, 1969. 
apparat erscheinen, verglichen mit den wechselseitigen Beziehungen der Zweige des repressıven Staatsapparatcs, mit mehr Unabhängigkcit ausgestattet.

Warum nun sollte man von idcologischen Stuatsapparaten sprechen, also die ideologisch-fundierten Institutionen in das staatliche System einbeziehen? [p. a.: r. Die Ideologie ist nicht etwas „Neutrales $\alpha$ in der Gesellschaft. Darüber hinaus ist festzustellen, daß politische Herrschaft nicht allein mittels physischer Repression ausgeübt werden kann. Sie bedarf des unmittelbaren und entschiedenen Einfluß des Ideologischen: in diesem Sinne ist das Ideologische, in seiner Erscheinungsform von ideologischen "Apparaten $\alpha$, unmittelbar ins staarliche System einbegriffen, das seinerscits Ausdruck, Garant und Ort der politischen Macht ist.]

I. Wenn der Staat ais die Instanz definiert wird, die den Zusammenhalt einer sozialen Formation aufrechterhält und die die Produkrionsbedingungen eines sozialen Systems durch Aufrechterhaltung der Klassenherrschaft reproduziert, so ist of fenkundig, daß die fraglichen Institutionen - die ideologischen Apparate des Staates - genau dieselbe Funktion erfüllen. [p. a.: 2. Auf die marxistische Staatsdefinition muß man sich wie folgt beziehen: Die Klassiker definieren den Staat nicht in erster Linie als Inhaber des Monopols repressiver physischer Gewalt, sondern durch seine gesellschaftliche Funktion als Instanz. Der Staat ist die zentrale Instanz, deren Funktion in der Aufrechterhaltung der Einheit und des Zusammenhalts einer gesellschaftlichen Formation besteht, in der Produktion der Reproduktionsbedingungen einer Formation insgesamt und somit, in der erweiterten Reproduktion der Produktionsbedingungen, in einem System von Klassenkampf. In einem solchen System liegt die Hauptrolle der ideologischen Apparate in der Erfüllung eben derselben Funktion. Die ideologischen Appazate haben als Hauptfunktion, den Zusammenhalt und die Einheit einer Formation aufrechtzuerhalten - die herrschende Ideologie skittet e eine Formation -, indem sie die Reproduktionsbedingungen einer Formation als Ganzer produzieren und so die Produktionsbedingungen reproduzieren - die Ideologie beschränkt sich nicht auf Ideen. Schon hier zeigt sich die Identität der Funktion des Staatsapparates im engeren Sinn und der der ideologischen Apparate.]

2. [p.a.: 3.] Der repressive Staatsapparat selbst ist in einer bestimmten Formation die Bedingung der Möglichkeit des Bestehens und Funktionierens dieser Institutionen oder ideologischen Apparate. Wenn auch ihre Rolle überwiegend eine ideologische ist und der repressive Staatsapparat im allgemeinen nicht unmittelbar in ihr Funktionieren eingreift, so steht er doch ständig hinter ihnen, verteidigt und sanktioniert sie und schreibr ihnen schließlich durch sein Auftreten ihr Verhalten vor. Die Studentenberwegung in Frankreich und anderswo kann das heute in puncto Schulen und Universitäten bezeugen.

3. [p.a.: 4.] Obwohl diese ideologischen Apparate untereinander und in ihrem Verhältnis zum repressiven Staatsapparat eine bemerkenswerte Autonomie besitzen, gehören sie zu demselben System wie dieser repressive Apparat. Jede wichtige Veränderung der Staatsform hat Rückwirkungen, nicht nur auf [p.a.: die Rolle und] das wechselseitige Verhälenis der $¥ Z$ weige" des repressiven Staatsapparates, sondern auch auf [p. a.: die Rolle und] das wechselseitige Verhältnis der ideologischen Staatsapparate zueinander und das zwischen diesen und dem repressiven Staatsapparat. [p. a.: Nichts zeigt das besser als die verschiedenen Formen, die der kapitalistische Staat bisher angenommen hat.] Man brauche gar niche den Extremfall des Faschismus zu nehmen, um diese These zu belegen: man braucht nur zu erwähnen, wie sich dic Rolle und Verhältnisse der Kirche, der Parteien, Verbände, Schulen, Medien, der Familie - sowohl zuein- 
ander als auch zum repressiven Staatsapparat - unter den verschiedenen snormalen «ntwicklungsformen des kapitalistischen Staates modifiziert haben.

4. [p.a.: s.] Schließlich ein letzter Grund: Nach marxistisch-leninistischer Theorie bedeutet eine sozialistische Revolution nicht nur einen Wechsel in der Staatsgewalt, sondern diese muß auch den Staatsapparat [ $p$. a.: einschließlich der ideologischen Apparate] zerbrechen, d. h. radikal verändern. Wenn man nun die idcologischen Apparate in das Konzept des Staates einbezieht, ist evident, warum die Klassiker des Marxismus - of nur implizit - für notwendig hielten, die These von der Zerstörung des Staates nicht nur auf den repressiven, sondern auch auf den ideologischen Staatsapparat anzuwenden: Kirche, Parteien, Verbände, Schulen, Medien, Familie.

Dies bedeuter angesichts der [p.a.: relativen] Autonomie der ideologischen Staatsapparate sicherlich nicht, daß sie - alle oder einzelne von ihnen - zur selben Zeit oder auf dieselbe Weise zerbrochen, werden müssen [p. a.: können] wie der repressive Staatsapparat. Es bedeutet, da $B$ die „Zerstörung « der ideologischen Staatsapparate die "Zerstörung des repressiven Staatsapparates zur Vorbedingung hat. Es ist also beispielsweise ein illusionärer Irrglaube, wenn eine gewisse zeitgenössische These es für möglich hält, hier und jetzt zur nZerstörung* der Universität in kapitalistischen Gesellschaften überzugehen. Es bedeutet aber auch, daß die Entwicklung der sozialistischen Gesellschaft nicht vollendet werden kann, wenn nur der repressive Staatsapparat "zerbrochen * wird, die ideologischen Staatsapparate aber unversehrt beibehalten, ohne weiteres übernommen und nur ihrer Funktion nach verändert werden.

Man sieht, mit dieser Frage nähern wir uns dem Problem der Diktatur des Proletariats und der Kulturrevolution; sie führe uns aber weg von Miliband. Ich habe niche die Absicht, hier die politischen Folgerungen aus Milibands Buch zu untersuchen, über die er sich übrigens weitgehend ausschweigt: die Frage bleibt offen. [...] 